\title{
Physical activity in persons with Parkinson disease: A feasibility study
}

\author{
C. Allyson Jones ${ }^{1 \#}$, Marguerite Wieler ${ }^{2 \S}$, Jennifer Carvajal ${ }^{1 \S}$, Logan Lawrence ${ }^{3 \S}$, \\ Robert Haennel ${ }^{1 \S}$ \\ ${ }^{1}$ Department of Physical Therapy, University of Alberta, Edmonton, Canada; ${ }^{\#}$ Corresponding Author: cajones@ualberta.ca \\ ${ }^{2}$ Faculty of Medicine and Dentistry, University of Alberta, Edmonton, Canada \\ ${ }^{3}$ Faculty of Physical Education and Recreation, University of Alberta, Edmonton, Canada
}

Received 3 September 2012; revised 7 October 2012; accepted 13 October 2012

\section{ABSTRACT}

Background: Physical activity for persons with Parkinson Disease (PD) is recommended yet little is known about the physical activity levels in this patient population. The primary aim was to assess the feasibility of using a direct measurement and self-report measure of physical activity in patients with PD. Methods: Physical activity was recorded in $\mathbf{1 1}$ out-patients with mild to moderate PD. An accelerometer based sensor system (SenseWear Pro Armband ${ }^{\mathrm{TM}}$ ) which was worn continuously over 2 days was used to measure physical activity. Minute by minute energy expenditure and steps per day were recorded. Self-report physical activity was measured using the Short QUestionnaire to ASsess Health-enhancing physical activity (SQUASH) which assessed average weekly activity. Results: Using the accelerometer based sensor system, $83 \%$ of the day was spent in sedentary activity with the majority active time spent at a light intensity (2.7 [SD 2.0] hrs/day). Self-reported mean number of hours for activities greater than 2.0 METs was 3.4 (SD 1.5) hrs/day. Although the overall time spent in activity did not differ between the accelerometer and SQUASH, participants reported a higher proportion of activities at the moderate and vigorous intensities than the accelerometer recorded. Conclusions: Measurement of physical activity is a challenge in persons with PD given the disease-related symptoms. We found that, by all accounts, a self-report measure of physical activity should be complemented with a direct measure of physical activity.

\footnotetext{
*Competing interests: the authors declare that they have no competing interests.

${ }^{\S}$ These authors contributed equally to this work.
}

Keywords: Parkinson Disease; Physical Activity; Quality of Life; Exercise Test

\section{BACKGROUND}

Parkinson Disease (PD) is a chronic progressive neurodegenerative disease characterized clinically by varying combinations of rest tremor, rigidity, bradykinesia, postural instability and gait disorder [1]. Impaired mobility together with "non-motor" features such as sleep and mood disturbance, autonomic dysfunction, and cognitive decline can lead to physical inactivity and chronic conditions associated with inactivity including osteoporosis and depression $[2,3]$.

Mounting evidence suggests that exercise may play a role in altering the progression of PD though its specific role in disease modification remains speculative at this point [4]. As a general principle, exercise is recommended for those with PD but daily activity level and cardiovascular response to exercise are not fully understood in this older patient population. Age-associated comorbidities, such as high blood pressure and muscle wasting, can decrease functional characteristics of exercise such as oxygen uptake $\left(\mathrm{VO}_{2}\right)$ [5]. While some findings suggest that persons with PD have peak aerobic capacity $\left(\mathrm{VO}_{2} \mathrm{max}\right)$ comparable to those of healthy adults $[6,7]$, others have reported reduced peak aerobic capacity and limited capacity to improve aerobic fitness in PD $[5,8,9]$.

Overwhelming epidemiological evidence supports the health benefits of regular physical activity in the general population such as improving muscle strength [10], increasing aerobic fitness while lowering energy expenditure, [11], and decreasing the risk of physical disability [12-14]. A direct relationship exists between the level of physical activity and health status with more physically active people at a lower risk for many chronic conditions [12]. Regardless of the health benefits of physical activity, prevalence rates of physical inactivity in the general 
population are in excess of $51 \%[12,15]$, with older persons less likely to be active [15].

Little attention has been directed toward the promotion of regular physical activity in persons with $\mathrm{PD}$, despite the knowledge that physical inactivity is a modifiable risk factor for many other chronic conditions such as cardiovascular disease, hypertension, diabetes mellitus, osteoporosis, obesity and depression [16]. Both age- and disease-related factors can compound the effect of inactivity in PD. Although the effectiveness of specific exercise regimens has been examined in PD [17,18], the extent to which persons with PD are physical active has not been extensively examined $[19,20]$. To evaluate the intensity of physical activity in this older patient population we undertook a feasibility study to evaluate direct and self-report measurements of physical activity. The purpose of a feasibility study is to provide direction in designing the main study. If we can identify the challenges of evaluating physical activity in patients with PD then a clearer basis of selection of outcome measurements can be utilized both in clinical trials and large epidemiological studies.

\section{METHODS}

A convenience sample of 11 PD outpatients was recruited between July 2009 and March 2010 at the Movement Disorders Program (Glenrose Rehabilitation Hospital in Edmonton, Alberta, Canada), which serves northern Alberta with a catchment area of approximately 1.5 million people. Inclusion criteria for the study consisted of the participant 1 ) having a primary diagnosis of PD as diagnosed by a movement disorders neurologist, 2) being untreated with PD medications at time of enrolment and 3) ambulating independently. All participants had Hoehn \& Yahr scores stage 1 or 2. Ethics approval was obtained from the University of Alberta Health Research Ethics.

Upon providing written informed consent, participants 1) completed a battery of self-report health-related quality of life measures, and 2) wore the SenseWear Pro Armband ${ }^{\mathrm{TM}}$ to estimate energy expenditure over 48 consecutive hours. To examine physical activity in terms of the intrinsic capacity to exercise we also asked participants to complete a treadmill test to evaluate aerobic fitness.

The self-administered measures consisted of sociodemographic data, such as date of birth, gender, marital status, and education, along with medical information (ambulatory status, comorbidities, and smoking history), and disease-related information (Hoehn \& Yahr stage [21], Unified Parkinson's Disease Rating Scale (UPDRS) [22], Activities-specific Balance Confidence (ABC) Scale [23]). Participants also completed a self-report measure of physical activity, the SQUASH [24], and health status,
Health Utilities Index Mark 3 (HUI3) [25-27].

\subsection{Assessment of Daily Physical Activity}

A direct measure of daily physical activity was estimated from the daily energy expenditure calculated from SenseWear Pro Armband ${ }^{\mathrm{TM}}$ (Body Media, Pittsburgh, PA) (SWA) data. The SWA accumulates data from a variety of sensors (including heat flux, biaxial accelerometer, galvanic skin response, skin temperature, near-body temperature) and combines these data with demographic characteristics (gender, height, weight, handiness and smoking status) to estimate energy expenditure using algorithms provided by the manufacturer [28-30]. The SWA also provides information on the number of steps/ day. This accelerometer based sensor system does not impede normal movement and can be worn throughout the day, in virtually all environments, except in water and for all types of activities, including sleep. The SWA has been shown to have good validity under both laboratory $[28,31,32]$ and free living conditions [33]; however, has not been validated in PD. There is an error rate of $8 \%$ with an intraclass correlation (ICC) of 0.80 for energy expenditure determined from the SWA and double labelled water [34].

The SWA was worn on the upper arm over the triceps muscle of the least affected side. For this study, participants were instructed to wear the SWA for three consecutive days so that 48 hours of near continuous data could be recorded. Participants were instructed to remove the device when bathing, showering or swimming. Once the SWA was returned to the lab, data were downloaded to generate the total time the device was worn, steps/minute, minute by minute energy expenditure and metabolic equivalent task (MET) intensity levels. One MET is the energy cost of resting quietly, often defined in terms of oxygen uptake as $3.5 \mathrm{~mL} /$ $\mathrm{kg} / \mathrm{min}$. Unlike other studies that have measured activity levels in PD using activity monitors [35,36], the intensity of activity using METs can be derived from the SWA.

Daily physical activity was also captured using a self-report measure of habitual physical activity, Short QUestionnaire to ASsess Health-enhancing physical activity (SQUASH) [24]. This instrument consists of four domains of which participants report on the frequency, duration, and intensity of different activities performed during the past "average" week. The activities include commuting activities (walking and bicycling), leisure time activities (walking, bicycling, gardening, odd jobs, and sports), household activities (light and heavy household chores), and work/school activities (light and heavy activities). The SQUASH assigns activities an intensity level based on the rate of energy expenditure which was expressed in METs [37]. The time per day for each domain is derived based on the reported frequency and du- 
ration.

The SQUASH has been evaluated in patient populations such as total joint arthroplasty, rheumatoid arthritis and lung transplant $[24,38,39]$. Overall, the SQUASH has comparable reliability as other self-report physical activity measures [40]. It also has reasonable validity when compared to an activity monitor in a sedentary population and patients with total hip arthroplasties [24, 40].

For both the self-report (SQUASH) and SWA data, sedentary was defined as values below 2.0 METS. Light activity was considered 2.0 - 2.9 METS, moderate activity 3.0 - 4.9 METS and vigorous activity as any value 5.0 METS or higher for those persons over 55 years of age. For the two participants who were under 55 years of age light activity was defined as $2.0-3.9$, moderate activity 4.0 to 6.4 and vigorous activity as MET values 6.5 or greater $[24,37]$. The total activity time for the SQUASH and SWA was calculated by summing the total time spent in each of the three activity categories.

\subsection{Assessment of Aerobic Fitness}

The intrinsic capacity to exercise was assessed using a submaximal exercise test on a treadmill. Participants were cleared to perform the exercise test by their neurologist and were screened using the Physical Activity Readiness Questionnaire [41]. Exclusion criteria for the assessment of aerobic fitness included 1) the use of cardiac medications or cardiac medical history, 2) a heart rate response at exercise onset corresponding to less than 10 beats/MET or 3 ) any physical limitation(s) that precluded walking on a treadmill. Aerobic fitness was estimated from physiological responses to three-four minute submaximal exercise loads performed on a motor driven treadmill (Woodway "Desmo" Model; DESMO-IED$220 \mathrm{~V})$. The initial workload involved walking at 2 or 3 $\mathrm{mph}$ with a $0 \%$ grade; subsequent workloads involved the same speed with a grade of $5 \%$ and $10 \%$ respectively. Because participants had mild to moderate PD (Hoehn \& Yahr Scale stage 1 and 2), they were deemed capable of safely performing treadmill walking [42]. Participants were asked to have refrained from consuming caffeine no more than five hours prior to testing.

During the five minute rest period prior to testing and throughout the exercise test, $\mathrm{VO}_{2}$ was recorded using a mobile metabolic cart (Oxycon Mobile, Jaegar Germany). Respiratory gases were recorded breath by breath using a mouth piece with nasal clips worn by the participant. Data were recorded for each breath and averaged over each one minute interval. Heart rate was also recorded for each one minute interval (Polar Electro, Finland) while blood pressure and Borg Scale of Perceived Exertion, a 15 point scale that quantifies the degree of exertion from none to exhaustion [43] was measured once during each stage. Aerobic fitness $\left(\mathrm{VO}_{2} \mathrm{max}\right)$ was estimated by extrapolating the heart rate (HR): $\mathrm{VO}_{2}$ responses through to the age predicted HRmax [44].

\subsection{Health Utilities Mark 3}

Health-related quality of life was assessed with the HUI3, a generic preference-based measure [25]. An advantage of a generic measure is that it provides for comparisons across different PD study cohorts and different conditions. The HUI3 was administered as a 15 -item questionnaire that assessed usual health status. Eight attributes are included in the HUI3 system: vision, hearing, speech, ambulation, dexterity, emotion, cognition and pain and discomfort, with five to six levels per attribute that range from highly disabled to no disability. Utility scores for single attributes range from 0.0 to 1.0 , with a score of 0.0 representing the lowest level of functioning on an attribute and a score of 1.0 representing full functional capacity. A difference of 0.05 in a single attribute is considered to be clinically important [26]. The overall HUI3 scores for health states range from -0.36 to $1.0(-0.36=$ worst possible health, $0.0=$ dead and $1.0=$ perfect health) [25]. Differences of 0.03 or more are considered to be clinically important [26]. The construct validity of the HUI provides support for its use in PD, in as much as the HUI is able to discriminate between clinically different groups of patients with PD, based on Hoehn \& Yahr stage and UPDRS motor scores [45].

\subsection{Analysis}

Energy expenditure was examined both as a continuous and categorical variable. For ease of interpretability, categorical data are presented. These categories were based on the Ainsworth's Compendium of Physical Activities [37]. Sedentary behavior refers to activities that do not increase energy expenditure substantially above the resting level and includes activities such as sleeping, sitting, lying down, and watching television, and other forms of screen-based entertainment. Operationally, sedentary behavior includes activities that involve energy expenditure of less than 2 METs. Light physical activity, which often is grouped with sedentary behavior but is in fact a distinct activity, includes activities such as slow walking, sitting and writing, cooking food, and washing dishes.

Descriptive statistics were used for all variables collected. The mean and standard deviation (SD) were reported for normally distributed data and the median and interquartile range (IQR) reported for data that were not normally distributed. To assess the significance of associations between categorical variables, $\chi^{2}$ tests were performed. Independent sample $t$-tests were used for normally distributed continuous variables. The agreement 
between the SWA and SQUASH times spent at the three activity levels: light, moderate and vigorous was visually inspected using Bland-Altman method [46].

All statistical testing was performed with two-tailed tests and at a 0.05 level of significance unless otherwise stated. Statistical analyses were performed using the SPSS $^{\circledR}$ software version 15.0.1 for Windows, SPSS Inc.

\section{RESULTS}

Eleven participants with mild to moderate PD between the ages of 48 and 71 years and who had a disease duration of 4.0 (SD 2.6) years were recruited. All participants were from the community and 8 were living with a spouse. All except one person were currently non-smokers. Participants walked independently: 3 participants could only walk 1 to 5 blocks, 6 reported the ability to walk more than 10 blocks. The major limitation to walking was fatigue as reported by participants $(n=5)$. All participants were categorized at a high level of mobility confidence by the ABC score (mean 90 SD 8 ). The mean number of comorbid conditions was 4.1 (SD 1.7) with mental health problems $(n=6)$ and chronic back pain $(n=5)$ being the most frequently cited conditions. The mean overall HUI3 score was 0.66 (SD 0.20). Dexterity (0.83) and pain (0.87) attributes had the lowest scores (Table 1).

The estimated $\mathrm{VO}_{2}$ max achieved from the treadmill test was 31.2 (SD 8.2$) \mathrm{mL} / \mathrm{kg} / \mathrm{min}$ with a range from 18 to $49 \mathrm{~mL} / \mathrm{kg} / \mathrm{min}$. The mean Borg exertion rating was 14 (SD 2). Four participants' $\mathrm{VO}_{2}$ max scores exceeded the $50^{\text {th }}$ percentile for their age and gender.

\section{Daily Physical Activity}

The participants wore the SWA an average of 23.3 (SD 0.7 ) hrs per day over the two days. Data from the SWA dual accelerometers recorded that participants averaged 5458 (SD 4416) steps/day.

When energy expenditures (METs) from the SWA were categorized, participants were sedentary for 19.4 (SD 3.8) hours/day or $83 \%$ of the total time recorded. Participants reported performing light intensity work an average of $2.7 \mathrm{hrs}$ (SD 2.0) hours/day or $79 \%$ of the time when they were active. Moderate activities accounted for $19 \%$ of their active time or a mean 1.1 (SD 1.8) hours/ day. The least amount of time when active was spent performing vigorous activities $(2 \%)$ with an average of 0.1 (SD 0.2) hours/day. The majority of participants $(\mathrm{n}=$ 7) reported no change in their routine activities when wearing the SWA; however, one participant reported increased activity level while wearing the SWA and another reported less activity while wearing the armband.

According to the SQUASH, the mean daily number of hours spent at any activity level greater than 2.0 METs
Table 1. Participant characteristics.

\begin{tabular}{ll}
\hline Characteristic & $\mathrm{n}=11$ \\
\hline Age (years), mean (SD) & $62.3(7.3)$ \\
Male, n (\%) & $7(64)$ \\
Education, $\mathrm{n}(\%)$ & \\
\hline Completed high school & $9(82)$ \\
Employment, $\mathrm{n}(\%)$ & \\
\hline Retired & \\
Working Full Time & $5(45)$ \\
Disability & $3(27)$ \\
Unemployed & $2(18)$ \\
Comorbid conditions, mean (SD) & $1(9)$ \\
Body Mass Index (kg/m²), mean (SD) & $4.1(1.7)$ \\
Current Smoker, $\mathrm{n}(\%)$ & $26.0(4.1)$ \\
Former Smoker, $\mathrm{n}(\%)$ & $1(9)$ \\
Years since diagnosis, median (IQR) & $6(58)$ \\
UPDRS (motor), mean (SD) & $4.0(1.1,6.5)$ \\
HUI3, mean (SD) & $17.6(10.2)$ \\
Vision & $0.87(0.08)$ \\
Hearing & $0.66(0.20)$ \\
Speech &
\end{tabular}

was $3.4 \mathrm{hrs}$ (SD 1.5). All participants reported activities in household (mean 0.9 [SD 0.5] hours/day) and leisure activities (mean 1.7 [SD 1.2] hours /day) with 8 reporting walking activities. Participants reported that $48 \%$ of this time was spent performing light activities, $38 \%$ at moderate intensity activities, and only $14 \%$ spent on vigorous activities.

The Bland-Altman plot (Figure 1) graphically depicts the comparison between the SWA and SQUASH. The wide intervals reflect the small sample size and the large variation of the differences. No differences were seen for the total number of minutes for active time (activities greater than 2 METs) between the SQUASH and SWA (p $=0.83$ ), yet participants reported greater time spent at moderate intensity and lesser time at light intensity than the SWA recorded. 

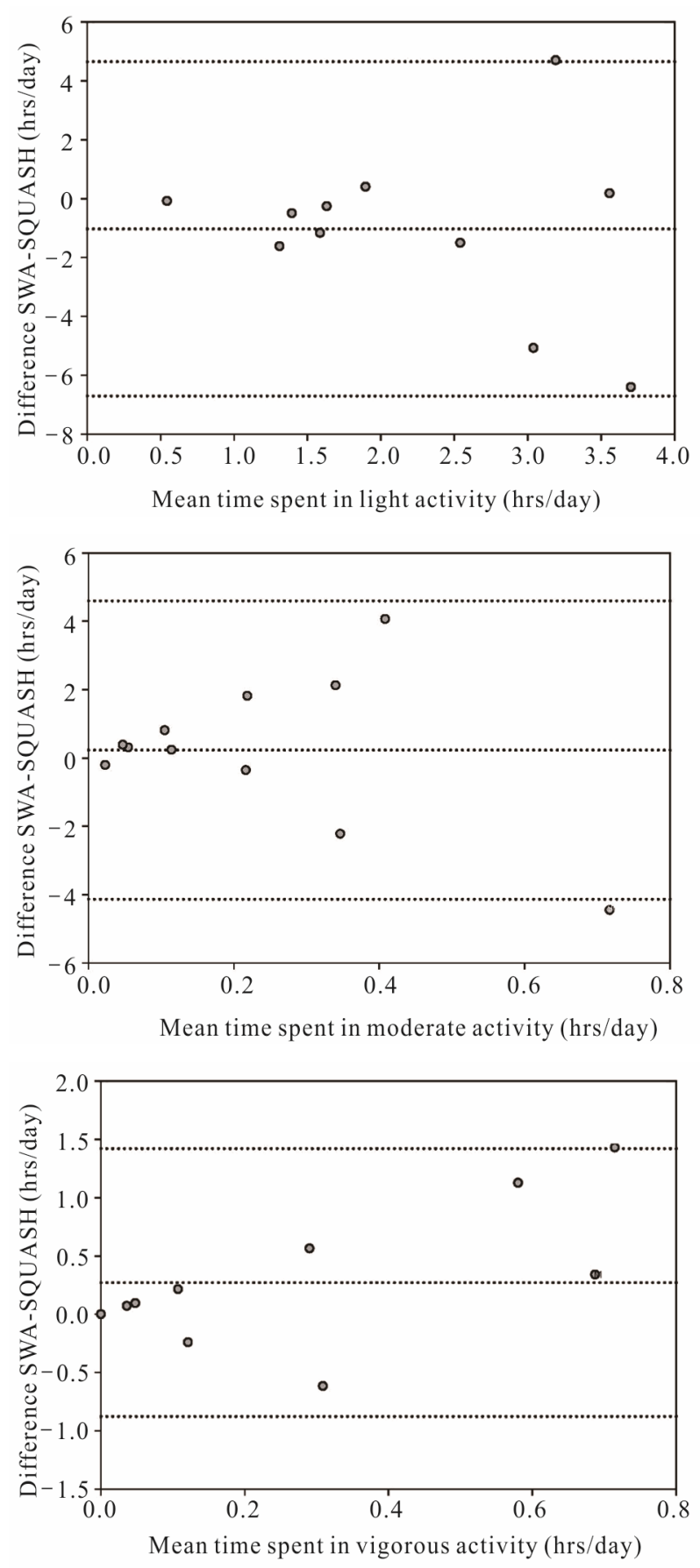

Figure 1. Difference between physical activity energy expenditure as measured by SenseWear Pro Armband ${ }^{\mathrm{TM}}$ (SWA) and SQUASH physical activity questionnaire plotted against the mean of the two measurements (Bland and Altman Plot). The upper and lower gridlines represent one standard deviation above and below the mean value.

\section{DISCUSSION}

To our knowledge there have been few studies that have examined physical activity level in persons with PD $[19,20]$. Because of the limited literature in this area, we conducted a feasibility study to identify measurement issues of physical activity in patients with PD. Overall, the measurement of physical activity in PD has been either self-report or direct measure [19,47] but rarely both types of measures have been used [20]. The findings from this feasibility study demonstrated that a physical activity measure needs to evaluate not only the active but the sedentary activities based on an overwhelming proportion $(83 \%)$ of time spent in sedentary activities. Participants tended to report a higher proportion of activities as moderate, whereas data from the SWA delineated these activities as light intensity. Data from the SWA and the SQUASH classified a small percentage of activity as vigorous. Inactivity was also reflected in $\mathrm{VO}_{2}$ max which was less than the $50^{\text {th }}$ percentile for seven participants.

Inactivity in this group was also exemplified by the number of daily steps. The number of steps recorded in these participants was typical of older adults and of those living with chronic conditions that average between 3500 and 5500 steps/day [48]. A lifestyle was defined as "sedentary" with less than 5000 steps/day; "low active" with a range of 5000 - 7499 steps/day; "somewhat active" with a range of 7500 - 9999 steps/day and 10,000 steps/day or more as "active" [49]. Applying these categories to the present data we observed that $59 \%$ of all the days monitored fell into the sedentary category whereas only 2 days met the criteria for "active" and those days were observed in a single participant. Further, on one of the "active" days the participant recorded over 21,000 steps; when this day was treated as an outlier the overall average for the group fell below the 5000 steps/day cut point (4712 SD 3626). Thus, the step data from the SWA suggests that the PD participants in this study were sedentary.

In spite of the inactivity, it is likely these participants were a relatively healthy cohort with minimal physical involvement as reflected by the Hoehn \& Yahr scores (stage 1 or 2) and the UPDRS motor scores which indicated mild to moderate disability. Based on our exclusion criteria it was unlikely that participants had symptomatic cardiac disease. While others have recognized that the fear of falling may limit activity [50], participants had a high confidence in their balance as reflected by the ABC scores. Moreover, they reported a higher level of healthrelated quality of life than reported in a communitybased cohort with PD. Health-related quality of life instruments such as the HUI3 can capture relevant information about patients with PD that would otherwise not be captured with the use of standard clinical scales or disease-specific health measures. The mean overall HUI3 score in our cohort was higher than a community-based cohort of persons with PD, 0.56 (95\%CI 0.48 to 0.63 ) [51] suggesting the burden of PD was less in this group of participants. Our findings are aligned with Ellis and 
colleagues who reported that personal factors rather than impairment were associated with exercise in the community for persons with PD [20].

The poor agreement seen between the SWA and the SQUASH suggests that a self-report measure of physical activity alone will not provide adequate detail of physical activity including sedentary activity. This agreement may have been affected by the SWA in that it has not been validated in PD, although participants in this study had mild PD. It was unlikely that tremor affected the readings from the SWA because only two participants had a tremor score greater than 0 on the UPDRS motor score for the least-affect upper limb side. These two participants had UPDRS tremor scores of 1-“slight or infrequently present" on the least affected side. The poor agreement between the two measures may be due, in part, to recall bias associated with the SQUASH values. It has been proposed by others that more intense activities are easier to recall than light ones [24]. Because this was an inactive group that did not perform significant amounts of vigorous activities, it may have been more difficult to recall light activities.

\section{CONCLUSION}

As with many chronic conditions, exercise is recommended for persons with PD [52], yet daily physical activity levels and patterns of participation remain relatively unknown in this patient population. Measurement of the intensity for physical activity is a challenge in persons with PD given the disease-related motor and non-motor symptoms. We found that, by all accounts, a self-report measure of physical activity should be complemented with a direct measure of physical activity. The capacity to exercise in terms of aerobic fitness should be taken into consideration when evaluating physical inactivity. Having established feasibility of the physical activity measures, we plan to identify physical activity levels and determinants of physical activity of PD in future largescale studies. Findings from studies that examine physical activity will provide direction to both clinical and health promotion interventions specifically designed for PD.

\section{ACKNOWLEDGEMENTS}

We wish to thank Dr. Martin Stevens for his assistance with the analysis of the SQUASH data.

Dr. Allyson Jones received salary support from the Alberta Heritage Foundation for Medical Research and the Canadian Institutes of Health Research.

\section{REFERENCES}

[1] de Lau, L.M. and Breteler, M.M. (2006) Epidemiology of
Parkinson's disease. Lancet Neurology, 5, 525-535. doi:10.1016/S1474-4422(06)70471-9

[2] Ravina, B., Elm, J., Camicioli, R., Como, P.G., Marsh, L., Jankovic, J. and Weintraub, D. (2009) The course of depressive symptoms in early Parkinson's disease. Movement Disorders, 24, 1306-1311. doi:10.1002/mds.22572

[3] Invernizzi, M., Carda, S., Viscontini, G.S. and Cisari, C. (2009) Osteoporosis in Parkinson's disease. Parkinsonism \& Related Disorders, 15, 339-346. doi:10.1016/j.parkreldis.2009.02.009

[4] Petzinger, G.M., Fisher, B.E., Van Leeuwen, J.E., Vukovic, M., Akopian, G., Meshul, C.K., Holschneider, D.P., Nacca, A., Walsh, J.P. and Jakowec, M.W. (2010) Enhancing neuroplasticity in the basal ganglia: The role of exercise in Parkinson's disease. Movement Disorders, 25, S141S145. doi: $10.1002 / \mathrm{mds} .22782$

[5] Garber, C.E. and Friedman, J.H. (2003) Effects of fatigue on physical activity and function in patients with Parkinson's disease. Neurology, 60, 1119-1124. doi:10.1212/01.WNL.0000055868.06222.AB

[6] Protas, E.J., Stanley, R.K., Jankovic, J. and MacNeill, B. (1996) Cardiovascular and metabolic responses to upperand lower-extremity exercise in men with idiopathic Parkinson's disease. Physical Therapy, 76, 34-40.

[7] Schenkman, M., Hall, D., Kumar, R. and Kohrt, W.M. (2008) Endurance exercise training to improve economy of movement of people with Parkinson disease: Three case reports. Physical Therapy, 88, 63-76. doi: $10.2522 /$ ptj.20060351

[8] Muller, T. and Muhlack, S. (2008) Impact of endurance exercise on levodopa-associated cortisol release and force increase in patients with Parkinson's disease. Journal of Neural Transmission, 115, 851-855. doi:10.1007/s00702-008-0018-7

[9] Haas, B.M., Trew, M. and Castle, P.C. (2004) Effects of respiratory muscle weakness on daily living function, quality of life, activity levels, and exercise capacity in mild to moderate Parkinson's disease. American Journal of Physical Medicine \& Rehabilitation, 83, 601-607. doi:10.1097/01.PHM.0000133436.61009.02

[10] Candow, D.G., Chilibeck, P.D., Facci, M., Abeysekara, S. and Zello, G.A. (2006) Protein supplementation before and after resistance training in older men. European Journal of Applied Physiology, 97, 548-556. doi:10.1007/s00421-006-0223-8

[11] Eldar, R., Marincek, C., Eldar, R. and Marincek, C. (2000) Physical activity for elderly persons with neurological impairment: A review. Scandinavian Journal of Rehabilitation Medicine, 32, 99-103. doi: $10.1080 / 003655000750045433$

[12] Warburton, D.E., Nicol, C.W. and Bredin, S.S. (2006) Health benefits of physical activity: The evidence. CMAJ, 174, 801-809. doi:10.1503/cmaj.051351

[13] Hamer, M., Chida, Y., Hamer, M. and Chida, Y. (2009) Physical activity and risk of neurodegenerative disease: A systematic review of prospective evidence. Psychological Medicine, 39, 3-11. doi:10.1017/S0033291708003681

[14] van den Brink, C.L., Picavet, H.S.J., van den Bos, G.A.M., 
Giampaoli, S., Nissinen, A. and Kromhout, D. (2005) Duration and intensity of physical activity and disability among European elderly men. Disability and Rehabilitation, 27, 341-347. doi:10.1080/09638280400018452

[15] Chen, J. and Millar, W.J. (1999) Health effects of physical activity. Health Reports, 11, 21-30.

[16] Bouchard, C., Shephard, R.J. and Stephens, T. (1994) Physical activity fitness and health: International proceedings and consensus statement. Human Kinetics, Champaign.

[17] Crizzle, A.M. and Newhouse, I.J. (2006) Is physical exercise beneficial for persons with Parkinson's disease? Clinical Journal of Sport Medicine, 16, 422-425. doi:10.1097/01.jsm.0000244612.55550.7d

[18] Baatile, J., Langbein, W.E., Weaver, F., Maloney, C. and Jost, M.B. (2000) Effect of exercise on perceived quality of life of individuals with Parkinson's disease. Journal of Rehabilitation Research and Development, 37, 529-534.

[19] Fertl, E., Doppelbauer, A. and Auff, E. (1993) Physical activity and sports in patients suffering from Parkinson's disease in comparison with healthy seniors. Journal of Neural Transmission-Parkinson's Disease and Dementia Section, 5, 157-161. doi:10.1007/BF02251206

[20] Ellis, T., Cavanaugh, J.T., Earhart, G.M., Ford, M.P., Foreman, K.B., Fredman, L., Boudreau, J.K. and Dibble, L.E. (2011) Factors associated with exercise behavior in people with Parkinson Disease. Physical Therapy, 91, 18381848. doi: $10.2522 / \mathrm{ptj} .20100390$

[21] Hoehn, M.M. and Yahr, M.D. (1967) Parkinsonism: Onset, progression and mortality. Neurology, 17, 427-442. doi:10.1212/WNL.17.5.427

[22] Fahn, S., Elton, R.L. and Members of the UPDRS Development Committee (1987) Unified Parkinson's disease rating scale. In: Fahn, S., Marsden, C.D., Goldstein, M. and Calne, D.B., Eds., Recent Developments in Parkinson's Disease, MacMillan Health Care Information, Florham Park, 153-163.

[23] Powell, L.E., Myers, A.M., Powell, L.E. and Myers, A.M. (1995) The Activities-specific Balance Confidence (ABC) scale. The Journal of Gerontology, Series A, Biological Sciences and Medical Sciences, 50A, M28-M34. doi:10.1093/gerona/50A.1.M28

[24] Wagenmakers, R., Akker-Scheek, I., Groothoff, J.W., Zijlstra, W., Bulstra, S.K., Kootstra, J.W.J., Wendel-Vos, G.C.W., van Raaij, J.J.A.M. and Stevens, M. (2008) Reliability and validity of the short questionnaire to assess healthenhancing physical activity (SQUASH) in patients after total hip arthroplasty. BMC Musculoskeletal Disorders, 9, 141. doi:10.1186/1471-2474-9-141

[25] Feeny, D., Furlong, W., Torrance, G.W., Goldsmith, C.H., Zhu, Z., Depauw, S., Denton, M. and Boyle, M. (2002) Multiattribute and single-attribute utility functions for the health utilities index mark 3 system. Medical Care, 40, 113-128. doi:10.1097/00005650-200202000-00006

[26] Horsman, J., Furlong, W., Feeny, D. and Torrance, G. (2003) The Health Utilities Index (HUI(R)): Concepts, measurement properties and applications. Health and Quality Life Outcomes, 1, 54. doi:10.1186/1477-7525-1-54
[27] Furlong, W., Feeny, D., Torrance, G.W., Goldsmith, C., Depauw, S., Boyle, M., Denton, M. and Zhu, Z. (1998) Multiplicative multi-attribute utility function for the Health Utilities Index Mark 3 (HUI3) system: A technical report, 98-11.

[28] Jakicic, J.M., Marcus, M., Gallagher, K.I., Randall, C., Thomas, E., Goss, F.L. and Robertson, R.J. (2004) Evaluation of the SenseWear Pro Armband to assess energy expenditure during exercise. Medicine \& Science in Sports \& Exercise, 36, 897-904. doi:10.1249/01.MSS.0000126805.32659.43

[29] Malavolti, M., Pietrobelli, A., Dugoni, M., Poli, M., Romagnoli, E., De, C.P. and Battistini, N.C. (2007) A new device for measuring resting energy expenditure (REE) in healthy subjects. Nutrition, Metabolism and Cardiovascular Disease, 17, 338-343.

[30] Cole, P.J., LeMura, L.M., Klinger, T.A., Strohecker, K., and McConnell, T.R. (2004) Measuring energy expenditure in cardiac patients using the Body Media Armband versus indirect calorimetry. A validation study. The Journal of Sports Medicine and Physical Fitness, 44, 262-271.

[31] Fruin, M.L. and Rankin, J.W. (2004) Validity of a multisensor armband in estimating rest and exercise energy expenditure. Medicine \& Science in Sports \& Exercise, 36, 1063-1069.

doi:10.1249/01.MSS.0000128144.91337.38

[32] King, G.A., Torres, N., Potter, C., Brooks, T.J. and Coleman, K.J. (2004) Comparison of activity monitors to estimate energy cost of treadmill exercise. Medicine \& Science in Sports \& Exercise, 36, 1244-1251. doi:10.1249/01.MSS.0000132379.09364.F8

[33] St-Onge, M., Mignault, D., Allison, D.B. and RabasaLhoret, R. (2007) Evaluation of a portable device to measure daily energy expenditure in free-living adults. The American Journal of Clinical Nutrition, 85, 742-749.

[34] Johannsen, D.L., Calabro, M.A., Stewart, J., Franke, W., Rood, J.C. and Welk, G.J. (2010) Accuracy of armband monitors for measuring daily energy expenditure in healthy adults. Medicine \& Science in Sports \& Exercise, 42, 2134-2140. doi:10.1249/MSS.0b013e3181e 0b3ff

[35] White, D.K., Wagenaar, R.C. and Ellis, T. (2006) Monitoring activity in individuals with Parkinson disease: A validity study. Journal of Neurologic Physical Therapy, 30, 12-21.

[36] Lim, I., van, W.E., Jones, D., Rochester, L., Nieuwboer, A., Willems, A.M., Baker, K., Hetherington, V. and Kwakkel, G. (2010) Does cueing training improve physical activity in patients with Parkinson's disease? Neurorehabilitation and Neural Repair, 24, 469-477. doi: $10.1177 / 1545968309356294$

[37] Ainsworth, B.E., Haskell, W.L., Whitt, M.C., Irwin, M.L., Swartz, A.M., Strath, S.J., O’Brien, W.L., Bassett, D.R., Jr., Schmitz, K.H., Emplaincourt, P.O., Jacobs, D.R., Jr. and Leon, A.S. (2000) Compendium of physical activities: An update of activity codes and MET intensities. Medicine \& Science in Sports \& Exercise, 32, S498-S504. doi:10.1097/00005768-200009001-00009

[38] Hurkmans, E.J., Maes, S., de, G.V., Knittle, K., Peeters, A.J., Ronday, H.K. and Vlieland, T.P. (2010) Motivation 
as a determinant of physical activity in patients with rheumatoid arthritis. Arthritis Care \& Research, 62, 371377. doi:10.1002/acr.20106

[39] Bossenbroek, L., ten Hacken, N.H., van der, B.W., Verschuuren, E.A., Koeter, G.H., de Greef, M.H. (2009) Cross-sectional assessment of daily physical activity in chronic obstructive pulmonary disease lung transplant patients. The Journal of Heart and Lung Transplantion, 28, 149-155. doi:10.1016/j.healun.2008.11.905

[40] Wendel-Vos, G.C., Schuit, A.J., Saris, W.H. and Kromhout, D. (2003) Reproducibility and relative validity of the short questionnaire to assess health-enhancing physical activity. Journal of Clinical Epidemiology, 56, 11631169. doi:10.1016/S0895-4356(03)00220-8

[41] Canadian Society for Exercise Physiology (2002) PAR-Q and you. http://uwfitness.uwaterloo.ca/PDF/par-q.pdf.

[42] Werner, W.G., DiFrancisco-Donoghue, J., Lamberg, E.M. (2006) Cardiovascular response to treadmill testing in Parkinson disease. Journal of Neurological Physical Therapy, 30, 68-73.

[43] Borg, G. (1970) Perceived exertion as an indicator of somatic stress. Scandinavian Journal of Rehabilitation Medicine, 2, 92-98.

[44] American College of Sports Medicine (2006) ACSM's Guidelines for Exercise Testing and Prescription. Lippincott Williams \& Wilkins, New York.

[45] Siderowf, A., Ravina, B. and Glick, H.A. (2002) Preference-based quality-of-life in patients with Parkinson's disease. Neurology, 59, 103-108. doi:10.1212/WNL.59.1.103

[46] Bland, J.M. and Altman, D.G. (1986) Statistical methods

\section{LIST OF ABBREVIATIONS}

ABC scale: Activities-Specific Balance Confidence Scale HR: Heart Rate

HUI3: Health Utilities Index Mark 3

ICC: Intra Class Correlation

MET: Metabolic Equivalent Task for assessing agreement between two methods of clinical measurement. Lancet, 1, 307-310. doi:10.1016/S0140-6736(86)90837-8

[47] Elbers, R., van Wegen, E.E., Rochester, L., Hetherington, V., Nieuwboer, A., Willems, A.M., Jones, D. and Kwakkel, G. (2009) Is impact of fatigue an independent factor associated with physical activity in patients with idiopathic Parkinson's disease? Movement Disorders, 24, 15121518. doi: $10.1002 / \mathrm{mds} .22664$

[48] Tudor-Locke, C. (2001) A preliminary study to determine instrument responsiveness to change with a walking program: physical activity logs versus pedometers. Research Quarterly for Exercise \& Sport, 72, 288-292.

[49] Tudor-Locke, C. and Bassett, D.R., Jr. (2004) How many steps/day are enough? Preliminary pedometer indices for public health. Sports Medicine, 34, 1-8. doi:10.2165/00007256-200434010-00001

[50] Pickering, R.M., Grimbergen, Y.A., Rigney, U., Ashburn, A., Mazibrada, G., Wood, B., Gray, P., Kerr, G. and Bloem, B.R. (2007) A meta-analysis of six prospective studies of falling in Parkinson's disease. Movement Disorders, 22, 1892-1900. doi: $10.1002 / \mathrm{mds} .21598$

[51] Pohar, S.L. and Jones, C.A. (2009) The burden of Parkinson Disease and concomitant comorbidities. Archives of Gerontology and Geriatrics, 49, 317-321. doi:10.1016/j.archger.2008.11.006

[52] Deane, K.H., Jones, D., Playford, E.D., Ben-Shlomo, Y., Clarke, C.E., Deane, K.H., Jones, D., Playford, E.D., Ben-Shlomo, Y. and Clarke, C.E. (2001) Physiotherapy for patients with Parkinson's disease: A comparison of techniques. Cochrane Database of Systematic Reviews, CD002817.

PD: Parkinson Disease

SD: Standard Deviation

SWA: SenseWear Pro Armband ${ }^{\mathrm{TM}}$

SQUASH: Short QUestionnaire to ASsess Health-Enhancing Physical Activity

UPDRS: Unified Parkinson's Disease Rating Scale 